\title{
Mixed Adenocarcinoid Tumor Presenting as Acute Appendicitis
}

\author{
Kalyani Regetia , Waqas Jehangir ${ }^{\mathrm{a}, ~ c}$, Shoaib Zafar ${ }^{\mathrm{a}}$, Shuvendu Sen ${ }^{\mathrm{a}}$, \\ Ibrahim Sidhom ${ }^{\mathrm{b}}$, Abdalla Yousif ${ }^{\mathrm{a}}$
}

\begin{abstract}
Mixed adenocarcinoid tumors are not uncommon neoplasms of appendix. The clinical presentation of these tumors is often similar to that of acute appendicitis or may present as asymptomatic. These tumors are found incidentally during histopathological examination of the resected appendix following appendectomy or other abdominal procedures. Mixed adenocarcinoids usually behave as adenocarcinomas with rapid metastasis, so prognosis depends upon how aggressive the tumor behaves. The present study reports a case of a 53-year-old male who presented with abdominal pain and fever for 1 day and underwent successful appendectomy and recovered later. Subsequently, a mixed adenocarcinoma with carcinoid features of the appendix was diagnosed by histopathological examination. Follow-up examination of the patient in 3 months revealed metastasis of carcinoma to the peritoneum with adenocarcinoma features.
\end{abstract}

Keywords: Appendicitis; Adenocarcinoids; Prognosis

\section{Introduction}

Appendix tumors are rare, contributing to only 1\% of appendectomy specimens and only about one half of $1 \%$ of intestinal neoplasms [1]. Among all, carcinoid tumors are the most common tumors contributing to over $50 \%$ of all tumors. Here we present a case of mixed features of tumor consisting of both carcinoid and adenocarcinoma (signet ring cell type) features which is a rare type; to the best of our knowledge, only few cases of these tumors have been reported so far. Adenocarcinoid is more aggressive than carcinoids but adenocarcinoid

Manuscript accepted for publication October 30, 2015

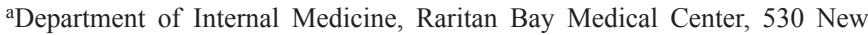
Brunswick Ave., Perth Amboy, NJ 08861, USA

${ }^{b}$ Department of Hematology \& Oncology, Raritan Bay Medical Center, 530 New Brunswick Ave., Perth Amboy, NJ 08861, USA

${ }^{\mathrm{c} C}$ Corresponding Author: Waqas Jehangir, Department of Internal Medicine, Raritan Bay Medical Center, 530 New Brunswick Ave., Perth Amboy, NJ 08861, USA. Email: wjehangir@hotmail.com

doi: http://dx.doi.org/10.14740/gr692e has worse prognosis compared to malignant carcinoid but better than appendiceal adenocarcinoma. Adenocarcinoids have lowest frequency of regional node metastasis but they have potential to spread intraperitoneally as described in the case below. Mixed feature of tumor consisting of carcinoid and adenocarcinoma is a rare type.

\section{Case Report}

A 53-year-old male presented with 1 day duration of acute severe right lower quadrant abdominal pain, sharp, non-radiating associated with fever, chills and nausea but no vomiting or weight loss. On physical exam, his vital signs were blood

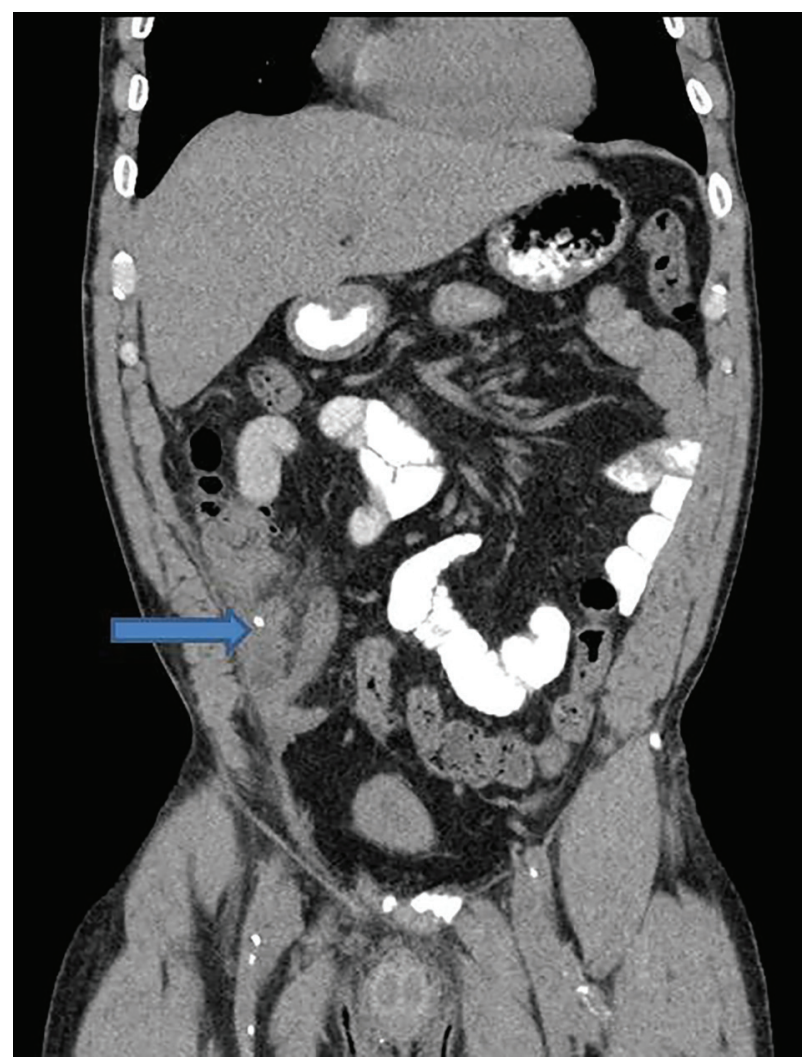

Figure 1. CT abdomen of pelvis showing appendicolith. 


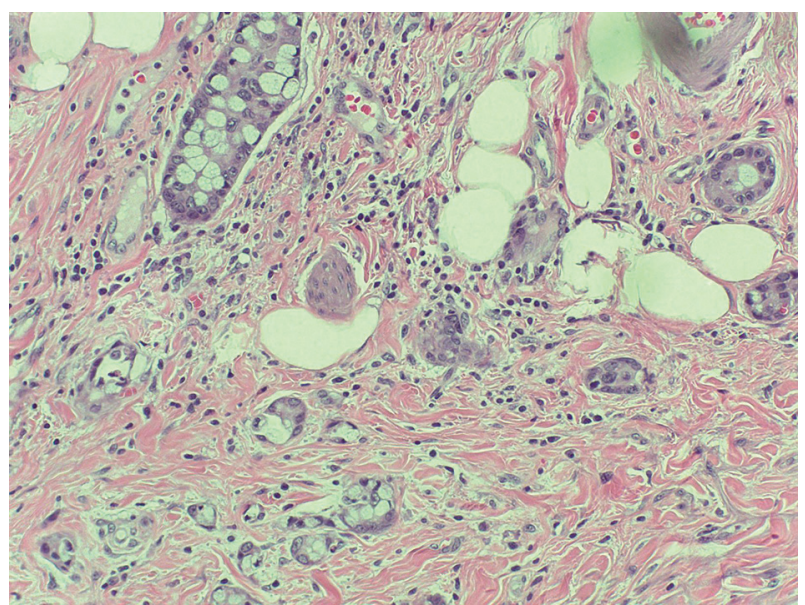

Figure 2. Mixed adenocarcinoid tumor.

pressure $126 / 68 \mathrm{~mm} \mathrm{Hg}$, pulse $90 / \mathrm{min}$, respiratory rate $14 / \mathrm{min}$, and temperature $99.4^{\circ} \mathrm{F}$. Abdominal exam revealed distension tenderness to Mcburney's point, with guarding and rigidity but bowel sounds were normal. Laboratory data showed hemoglobin $13.4 \mathrm{~g} / \mathrm{dL}$, hematocrit $40.1 \%$, white count $8.1 \times 10^{3} /$ $\mu \mathrm{L}$, platelets $172 \times 10^{3} / \mu \mathrm{L}$, sodium $133 \mathrm{mmol} / \mathrm{L}$, potassium $4.6 \mathrm{mmol} / \mathrm{L}$, chloride $99 \mathrm{mmol} / \mathrm{L}$, bicarbonate $20 \mathrm{mmol} / \mathrm{L}$, BUN $24 \mathrm{mg} / \mathrm{dL}$, creatinine $2.1 \mathrm{mg} / \mathrm{dL}$ and glucose $181 \mathrm{mg} / \mathrm{dL}$. Computed tomography (CT) scan of abdomen showed the perforated appendicitis with two appendicoliths seen in the right lower quadrant (Fig. 1). Laparoscopic appendectomy with drainage of periappendiceal abscess was performed. Surgical pathology of appendix showed moderately differentiated adenocarcinoma with signet-ring cell $(<50 \%$ signet ring component) and carcinoid features involving muscular wall (Fig. 2). Tumor was penetrating and perforating the visceral serosa and diffusely infiltrating $>4 / 5$ wall of the proximal portion of appendix with lymphovascular invasion. Special studies showed that the tumor is immunoreactive to CK20 (Fig. 3), focal to CD56, chromogranin (Fig. 4), synaptophysin; negative to CK7 (Fig. 5), TTF-1, PSA and CDx2. Mucin stain is positive (Fig.

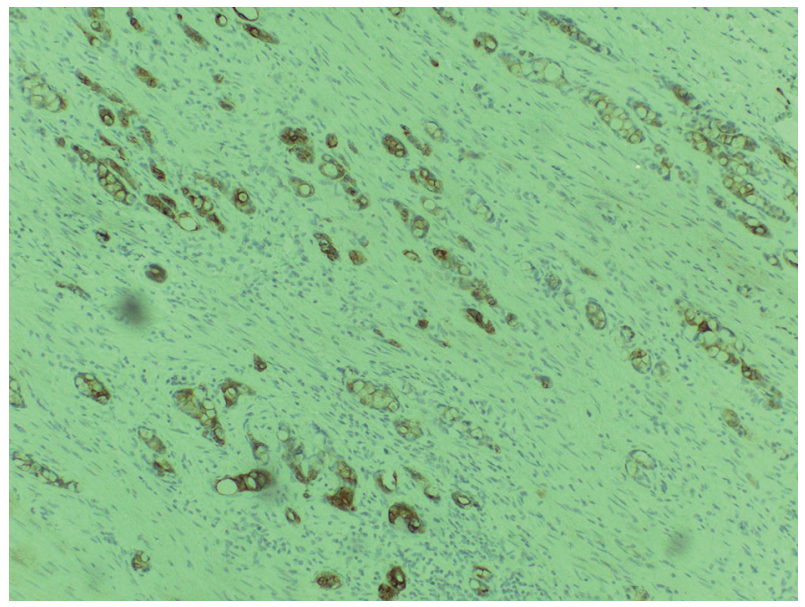

Figure 3. CK20 positive staining.

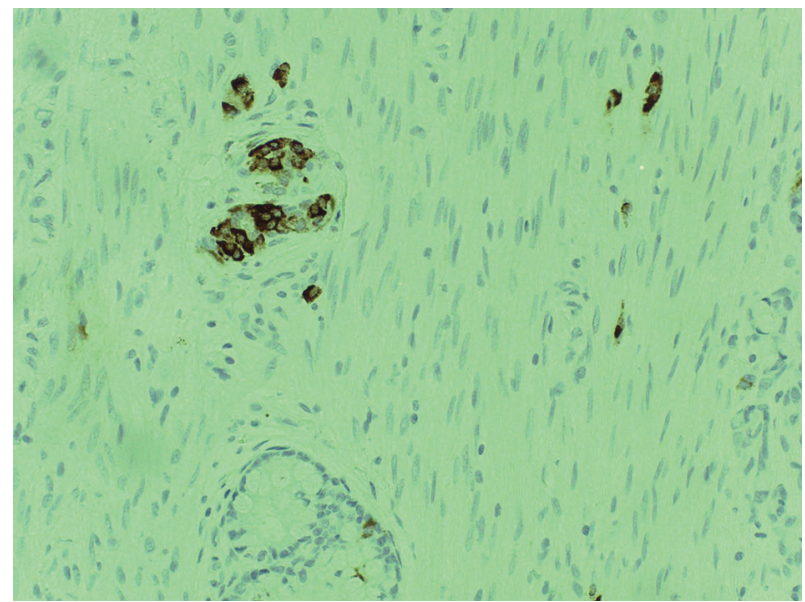

Figure 4. Chromogranin positive stain.

6). The immunostains support the diagnosis. Patient was started on chemotherapy and after 3 months, patient complained of abdominal pain for which $\mathrm{CT}$ abdomen was performed which showed findings suspicious for spreading cancer. Patient failed chemotherapy and underwent hemicolectomy and peritonectomy as there were seedings on the peritoneum.

\section{Discussion}

The existence of mixed adenoendocrine patterns in digestive system has been well documented. The first description date back in 1924 was reported [2]. A great number of these cases have been reported in appendix [3], and most of the cases are reported as adenocarcinoids/goblet cell carcinoids [3]. Edmonds et al [4] reported the largest series of 86 cases of goblet cell carcinoid of the appendix. Carr and Sobin [5] described 13 cases of dual carcinoid/epithelial neoplasia occurring in the appendix. Jain and Das [6] described a collision tumor involving the terminal ileum and cecum. To our knowledge, only few cases of collision tumor have been reported and the one

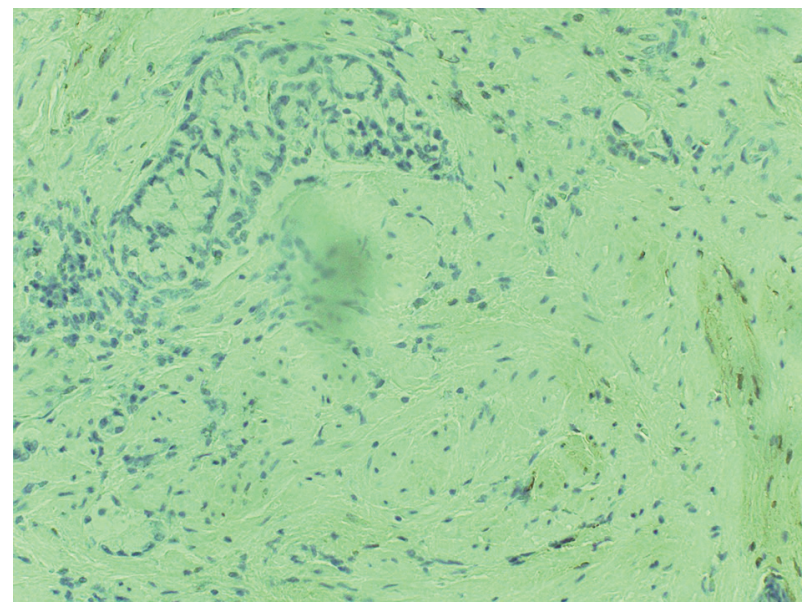

Figure 5. CK7 negative staining. 


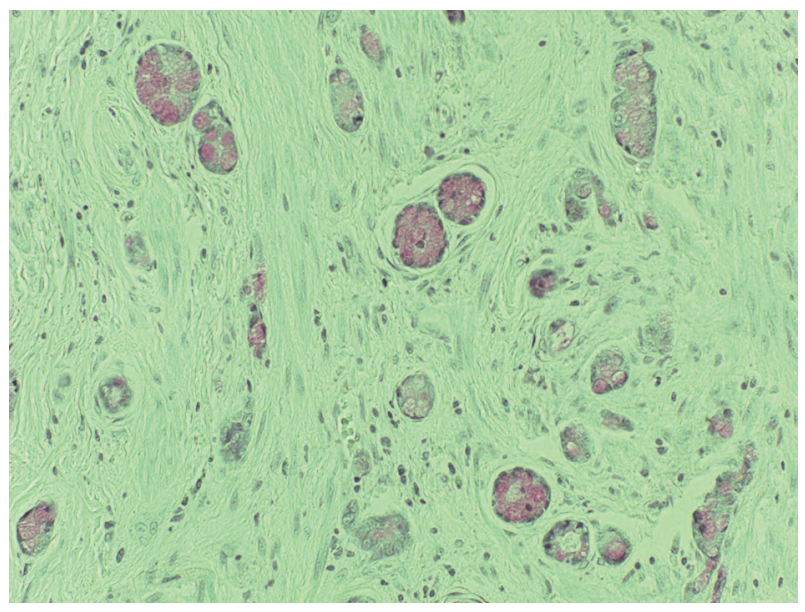

Figure 6. Mucin positive staining.

reported by Rossi et al [7] is adenocarcinoma associated with small cell carcinoma.

The histology of composite/collision tumors is not well understood despite the embryonic origin of composite tumors [8] is considered to be derived from a single event in which the initial neoplastic clone undergoes divergent differentiation, while collision tumors are considered to be of biclonal derivation, resulting from two separate but adjacent neoplasms [8]. However, this classification is not universally accepted since the cell of origin is still not very clear [2].

Histopathologically, collision tumors usually do not show transition [9], although intimately juxtaposed not intermixed. Single tumor component is observed at metastasis site; on the other hand, composite has both glandular and carcinoid elements in the metastasis site and also endocrine and epithelial cells are intermixed in same tissue and transitions are observed [9].

It is important to differentiate the type of tumor because of the difference in clinical behavior. Localized carcinoid tumors have excellent prognosis with 5-year survival approaching $99 \%$. Some cases of goblet cell carcinoid or adenocarcinoma were reported to be of low malignancy. But the majority of non-localized cases reported a malignancy of intermediate grade. However, predicting the prognosis of either collision or composite tumors still remains a matter of debate. Contrary to former belief, composite tumors do not have a better prognosis than ordinary adenocarcinoma $[10,11]$. In the present case, we described a case of collision tumor with features of both adenocarcinoma (signet ring cells) and carcinoid with metastasis to peritoneum with adenocarcinoma elements. And also patient had failed chemotherapy and finally ended up with hemicolectomy and peritonectomy.

\section{Conclusion}

Treating a case of collision or composite tumors is really challenging. In the above case, we described a patient with collision tumor who presented with appendicitis and had appendectomy with failed chemotherapy, and ultimately ended up with hemicolectomy and peritonectomy with ileostomy. This tumor had aggressive clinical course and behaved like adenocarcinoma than carcinoid.

\section{Author Contributions}

$\mathrm{KR}$, WJ and SZ drafted the manuscript. SS, IS and AY coordinated and helped to draft the manuscript. All authors read and approved the final manuscript.

\section{Funding}

None.

\section{Conflict of Interest}

This is to state that there has been no activity or involvements that will raise the question of bias in this case report or any of the conclusions or opinions that it stands for.

\section{References}

1. Hesketh KT. The management of primary adenocarcinoma of the vermiform appendix. Gut. 1963;4:158-168.

2. Cordier R. Les cellules argentaffines dans les tumerus intestinales. Arch Int Med Exp. 1924;1:59-63.

3. Levendoglu H, Cox CA, Nadimpalli V. Composite (adenocarcinoid) tumors of the gastrointestinal tract. Dig Dis Sci. 1990;35(4):519-525.

4. Edmonds P, Merino MJ, LiVolsi VA, Duray PH. Adenocarcinoid (mucinous carcinoid) of the appendix. Gastroenterology. 1984;86(2):302-309.

5. Carr NJ, Sobin LH. Epithelial noncarcinoid tumors and tumor-like lesions of the appendix. Cancer. 1995;76(11):2383-2384.

6. Jain M, Das KK. Concurrent occurrence of terminal ileum carcinoid tumor and cecal adenocarcinoma: a collision tumor or composite tumor? Indian J Pathol Microbiol. 2009;52(2):285-286.

7. Rossi G, Bertolini F, Sartori G, Bigiani N, Cavazza A, Foroni M, Valli R, et al. Primary mixed adenocarcinoma and small cell carcinoma of the appendix: a clinicopathologic, immunohistochemical, and molecular study of a hitherto unreported tumor. Am J Surg Pathol. 2004;28(9):1233-1239.

8. Goldenberg DM, Fisher ER. Histogenetic relationship between carcinoids and mucin-secreting carcinomas of colon as revealed by heterotransplantation. Br J Cancer. 1970;24(3):610-614.

9. Lewin K. Carcinoid tumors and the mixed (composite) glandular-endocrine cell carcinomas. Am J Surg Pathol. 1987;11(Suppl 1):71-86.

10. Rogers LW, Murphy RC. Gastric carcinoid and gastric carcinoma. Morphologic correlates of survival. Am J 
Surg Pathol. 1979;3(3):195-202.

11. Murayama H, Imai T, Kikuchi M. Solid carcinomas of the stomach. A combined histochemical, light and electron microscopic study. Cancer. 1983;51(9):1673-1681. 